\title{
Elderly Taiwanese who spend more on fruits and vegetables and less on animal-derived foods use less medical services and incur lower medical costs
}

\author{
Yuan-Ting C. Lo ${ }^{1}$, Mark L. Wahlqvist ${ }^{1,2,3}$, Yi-Chen Huang ${ }^{4}$ and Meei-Shyuan Lee ${ }^{1,3 *}$ \\ ${ }^{1}$ School of Public Health, National Defense Medical Center, Taipei 11490, Taiwan, ROC \\ ${ }^{2}$ Institute of Population Health Sciences, National Health Research Institutes, Miaoli County 35053, Taiwan, ROC \\ ${ }^{3}$ Monash Asia Institute, Monash University, Caulfield East, VIC 3145, Australia \\ ${ }^{4}$ National Defense Medical Center, Graduate Institute of Life Sciences, Taipei 11490, Taiwan, ROC \\ (Submitted 28 August 2015 - Final revision received 11 November 2015 - Accepted 23 November 2015)
}

\section{Abstract}

A higher intake of fruits and vegetables (F\&V) compared with animal-derived foods is associated with lower risks of all-cause-, cancer- and CVD-related mortalities. However, the association between consumption patterns and medical costs remains unclear. The effects of various food group costs on medical service utilisation and costs were investigated. The study cohort was recruited through the Elderly Nutrition and Health Survey in Taiwan between 1999 and 2000 and followed-up for 8 years until 2006. It comprised free-living elderly participants who provided a 24-h dietary recall. Daily energy-adjusted food group costs were estimated. Annual medical service utilisation and costs for 1445 participants aged 65-79 years were calculated from the National Health Insurance claim data. Generalised linear models were used to appraise the associations between the food group costs and medical service utilisation and costs. Older adults with the highest F\&V cost tertile had significantly fewer hospital days (30\%) and total medical costs (19\%), whereas those in the highest animal-derived group had a higher number of hospital days (28\%) and costs (83\%) as well as total medical costs (38\%). Participants in the high F\&V and low animal-derived cost groups had the shortest annual hospitalisation stays $(5.78 \mathrm{~d}$ ) and lowest costs (NT\$38600) as well as the lowest total medical costs (NT\$75800), a mean annual saving of NT $\$ 45200 /$ person. Older adults who spend more on F\&V and less on animal-derived foods have a reduced medical-care system burden. This provides opportunities for nutritionally related healthcare system investment strategies.

\section{Key words: Fruits and vegetables: Animal-derived foods: Food costs: Medical costs}

The health benefits of fruit and vegetable $(\mathrm{F} \& \mathrm{~V})$ consumption are widely recognised. A high $F \& V$ intake has been associated with low risks of hypertension ${ }^{(1)}, \mathrm{CHD}^{(2)}$, ischaemic heart disease ${ }^{(3)}$ and mortality (all-cause, cancer and CVD) ${ }^{(4-6)}$ in community-dwelling elderly people. Conversely, high consumption of animal-derived food, such as red meat, particularly processed meat, is associated with a high risk of all-cause mortality ${ }^{(7-9)}$.

Food security is a growing concern worldwide, and low-income households may face situations in which food or money is depleted. The global action plan for the prevention and control of non-communicable diseases (2013-2020) emphasises food availability, affordability and sustainability, contingent on optimising F\&V consumption ${ }^{(10)}$. Food price is a major determinant of food choice ${ }^{(11)}$, and food expenditure correlates positively with a high intake of F\&V and the associated dietary quality ${ }^{(12)}$.

The Taiwan National Health Insurance (NHI) is a compulsory programme launched in 1995; however, certain healthcare-related problems and challenges persist, particularly incremental ageing and non-communicable diseases ${ }^{(13)}$. Personal healthcare expenditure for people aged 60 years or older is 2-3-fold that of the general population ${ }^{(14)}$. With ageing and disability attributable to non-communicable diseases, healthcare costs can further increase unless affordable preventive measures are taken.

Household or individual spending more on $\mathrm{F} \& \mathrm{~V}$ rather than animal-derived foods has been associated with a reduced mortality risk for children under the age of 5 years in Indonesia ${ }^{(15)}$ and for older adults in Taiwan ${ }^{(16)}$. This suggests that F\&V expenditure has health outcome relevance across age groups. To our knowledge, the relationship between food group costs and those of the medical-care system is yet to be explored. Nutritional-economic approaches to address health outcomes are required, particularly for vulnerable groups such as children, reproductive-age women, older adults and low-income families. We have prospectively studied the effects of food group costs on medical service utilisation and costs in a nation-wide study in Taiwan of older adults.

\section{Methods}

\section{Study participants}

Free-living participants aged 65 years or older were recruited to a nation-wide representative Elderly Nutrition and Health Survey in Taiwan (NAHSIT) between 1999 and 2000. The study design has 
been published elsewhere ${ }^{(17)}$. We interviewed 1911 participants through a home-based survey, which included a 24-h dietary recall. Trained interviewers used several tools such as food-piece models, abstract food models, measuring cups, spoons, electronic weighing and cue-cards to enquire about foods and dishes consumed in the past $24 \mathrm{~h}$. In order to cross-validate the recall data, we also involved other members of the household ${ }^{(18)}$. To ensure the representativeness of the estimates for an individual diet, 126 participants with unreasonably high or low total daily energy intakes $(<2092 \mathrm{~kJ} / \mathrm{d}(<500 \mathrm{kcal} / \mathrm{d})$ or $>14644 \mathrm{~kJ} / \mathrm{d}(>3500 \mathrm{kcal} / \mathrm{d})$ in women; $<3347 \mathrm{~kJ} / \mathrm{d}(<800 \mathrm{kcal} / \mathrm{d})$ or $17573 \mathrm{~kJ} / \mathrm{d}(>4200 \mathrm{kcal} / \mathrm{d})$ in men $)^{(19)}$ and two participants on a liquid diet were excluded. The remaining participants were linked to the National Death Registry and NHI claim data up to 31 December 2006 (8-year follow-up), through their unique personal identification number. Of these, 133 participants had inaccurate death records, missing NHI records because of an incorrect personal identification number or a catastrophic illness before the interview; therefore, they were excluded. We did not wish to entertain the notion that high technology approaches and costs associated with terminal illness would be food sensitive. Participants who were older or died in the 1st year of follow-up might have had a prolonged illness or disease that manifested before death; these participants would have tended to use more medical services than others ${ }^{(20)}$. Therefore, analysis was performed by further excluding participants who died in the 1st year of follow-up and those aged over and equal to 80 years (1445 participants left) (Fig. 1).

\section{Ethics}

The present study was approved by the ethics committees of both Academia Sinica and the National Health Research Institutes in Taiwan. All the participants provided their written informed consent.

\section{Daily food group costs}

Food retail prices between January 1999 and December 2000 were compiled using several databases from the Taiwanese Council of Agriculture - namely, the Agriculture and Food Agency, Poultry and Livestock Products Current Trade and the Fisheries Agency ${ }^{(21-23)}$. The national average monthly prices (New Taiwan dollars, NT\$) for 843 foods (as purchased) were used to calculate the food costs at the interview dates. Prices were supplemented and adjusted according to the national supermarket chain price consistency or fluctuations throughout Taiwan in 2009. Supermarket food prices were discounted and deflated according to the consumer price index (CPI) for 1999-2000. Aggregate food costs (NT\$/d) were calculated by multiplying the purchased weight $(\mathrm{g})$ by the price of that particular food (NT\$/g) and summing the cost of each food group or all foods an individual had consumed as per the 24-h dietary recall. We assumed that the participants ate at home, and no adjustments were made for eating out at restaurants, for personal celebrations or for festive occasions according to the Chinese or other calendar. We have published the food price calculations in detail $^{(12)}$. In 1999-2000, US\$ 1 equalled NT\$31.

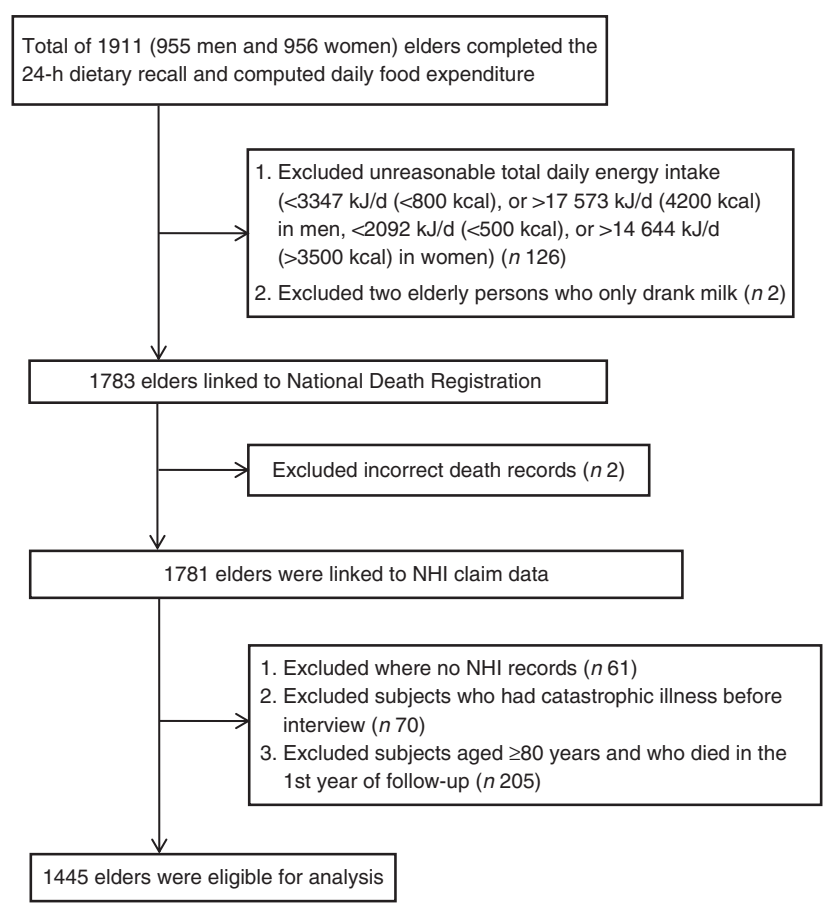

Fig. 1. Flow chart for participant selection. NHI, National Health Insurance.

All the food items were classified into four groups: F\&V comprising vegetables including fresh beans, fruits and fresh fruit juices; animal-derived foods comprising chicken, duck, pork, beef, goat, finfish, shellfish, crustaceans, eggs and dairy products; grain-based foods comprising rice and rice products, wheat and flour products, starchy roots and stems and dry beans; and miscellaneous foods and snacks except noodles and steamed buns. Thus, four food group costs were derived. Energy-adjusted food group costs were obtained using the residual method ${ }^{(24)}$ and assigned to tertiles $(\mathrm{T})$.

\section{National Health Insurance database}

The NHI is a universal, compulsory health insurance programme financed by a means-related premium system launched in 1995 , covering $>99 \%$ of the residents of Taiwan ${ }^{(25,26)}$. The National Health Insurance Research Database (NHIRD) provides claim data for reimbursement under the NHI. The NHIRD maintains complete beneficiary records comprising the clinical diagnoses (according to the International Classification of Diseases, Ninth Revision, Clinical Modification codes), catastrophic illnesses, medical costs and prescription claims associated with all outpatient and inpatient care ${ }^{(27)}$.

\section{Medical service utilisation and costs}

Medical service utilisation and costs were obtained from the NHI database. Both ambulatory care and hospital care utilisation and costs were considered and totalled. Ambulatory care included outpatient services, preventive care, dental services and emergency visits. Hospitalisation days were inpatient length of stay. Costs accounted for all resource inputs including those of 
medical and surgical treatments and contingent service fees. Medical data from the NHI claim data were collected longitudinally from the 1999-2000 interview date until the date of death or 31 December 2006. The average annualised utilisation and costs were obtained by dividing the total by the follow-up years for each participant. We assigned successive annual medical costs a yearly discount rate of $3 \%$, based on a yearly core CPI adjustment $^{(28)}$. For reasons presented under study participants, medical services or incurred medical costs within 12 months of death were excluded.

\section{Covariates}

The potential covariates were demographic factors, socio-economic status (SES), multi-morbidity, physical function, physical activity, sleeping quality, chewing ability and nutritional factors $^{(12,28-32)}$. Responses were recorded using personal interviews, a personally administered questionnaire at baseline. Demographic and SES factors recorded were sex, age (years), regions, ethnicity (Fukienese, Hakka, mainlander and indigenous), education level (illiterate, primary and lower, secondary education and higher), living arrangement, self-rated financial status and household income (<15000, 15000-29999, 30000-49999, $\geq 50000 \mathrm{NT} \$$ /month). Health status was perceived as good, fair or poor. Physical function was documented on the basis of activities of daily living (ADL). A total of nine questions regarding self-care task difficulty were asked, which included eating, transfer between bed and chair, walking indoors/outdoors, dressing, bathing, toileting and urinary/bowel continence. Everyday tasks can reflect physical health and living independence ${ }^{(33)}$. One or more of these difficulties was considered a significant limitation in physical function $^{(34)}$. Types and duration of sport and leisure time activities per week were obtained by the questionnaire, and daily physical activity as the number of metabolic equivalents (MET) was estimated. We classified physical activity into three levels $(<1 \cdot 5$, $1 \cdot 5-3,>3 \mathrm{MET} / \mathrm{d})^{(34)}$. Sleeping quality was assessed using the following question: 'How would you rate your overall sleeping quality in the past 3 months?'. The response was good, fair and poor $^{(35)}$. Self-reported chewing ability was assessed using the following question: 'Do you have difficulty chewing food?'. The response was either yes or no. For considering multi-morbidity, we used the Charlson co-morbidity index ${ }^{(36)}$ derived at baseline (1999 NHI claim data). Dietary quality was determined according to the 24-h recall and expressed as a dietary diversity score (DDS, range $0-6)^{(37)}$.

\section{Statistical analysis}

The mean and median of medical service utilisation and cost data are reported because their distributions are skewed to the right. Categorical variables were compared using $\chi^{2}$ tests. Generalised linear models (GLM) with a log link and $\gamma$ distribution were used to identify the relationships between food group costs (tertiles, T) and medical costs without and with potential covariates while being simultaneously adjusted for total food, vegetable and fruit and animal-derived foods costs. For medical service utilisation, GLM with a log link and Poisson distribution were used ${ }^{(38)}$. The cost for respondents who spent no money on medical costs was substituted using NT\$0.01. We used SAS, version 9.2 (SAS Institute Inc.) for all analyses. A two-tailed $P<0.05$ was considered to be statistically significant.

\section{Results}

Participants were followed-up for up to 8 years. Elderly participants whose F\&V costs were in the T2 or T3 ranges had significantly lower annual average emergency (visits and costs), hospitalisation (days and costs) and total medical costs but had higher outpatient services and dental services (visits) compared with those in T1. In contrast, those whose animal-derived food cost was in the $\mathrm{T} 2$ or $\mathrm{T} 3$ range had significantly higher annual average emergency (visits), hospitalisation (days) and total medical costs compared with those in T1. The mean total medical costs for increasing tertiles of F\&V costs were NT\$67500, NT\$46100 and NT\$49500; for increasing tertiles of animal-derived food costs, the costs were NT\$50 000, NT\$55 500 and NT\$57500. We focused on F\&V and animal-derived food costs associated with medical service utilisation and costs on outpatient services, emergency, hospitalisation and total medical costs (Table 1).

Older adults who spent less on F\&V (T1) were indigenous, illiterate, of low financial status or of poor health status or they had poor ADL, unsatisfactory chewing ability or a low DDS $(P<0 \cdot 001)$ (Table 2).

Table 3 lists the multivariable models and sensitivity analyses. No statistically significant differences were observed for emergency visits and costs. However, food group costs (F\&V and animal-derived) and outpatient services (visits) and hospitalisation (days and costs) were significantly associated. In model 2, elderly participants in the T3 F\&V food cost group had significantly fewer hospitalisation days (30\%) and total medical costs (19\%) but higher outpatient services (13\%) compared with those in T1 after controlling for all potential covariates. For animal-derived food costs, elderly participants in the $\mathrm{T} 3$ group had significantly more outpatient services (7\%), hospitalisation days (28\%), hospitalisation costs (83\%) and total medical costs (38\%) compared with those in the T1 group.

Fig. 2 illustrates the annual adjusted mean of inpatient care (days and costs) according to the tertiles of F\&V and animalderived food costs after covariates were controlled for. Elderly participants with F\&V food costs in T1 had the longest inpatient stays (11.5d) and the highest costs (NT\$88400) as well as total medical costs (NT\$116000) compared with those in T2 and T3. However, participants with animal-derived food costs in T1 had the shortest mean inpatient stays $(7.99 \mathrm{~d})$ and the lowest costs (NT\$42 200) as well as total medical costs (NT\$81 500).

We further examined, by combination, the simultaneous F\&V and animal-derived food costs to explore the consumption pattern that could lower the medical-care burden. For inpatient care, older adults with F\&V costs in $\mathrm{T} 2$ and $\mathrm{T} 3$ and those with animal-derived food costs in T1 had the shortest annual adjusted mean stays (5.78 d) and lowest hospitalisation costs (NT\$38600) as well as the lowest total medical costs (NT\$75800). Compared with the combined F\&V (T1) and animal-derived food (T1) costs, the mean difference in annual total medical costs for $\mathrm{F} \& \mathrm{~V}$ (T2 and $\mathrm{T} 3$ ) and animal-derived food (T1) was NT\$45200 (Fig. 3). 
Table 1. Annual medical service utilisation and costs by tertile (T) of daily food group costs ( $n$ 1445)

(Mean values and medians)

\begin{tabular}{|c|c|c|c|c|c|c|c|c|c|c|c|c|c|c|c|c|c|c|c|c|c|c|c|c|}
\hline \multirow{4}{*}{$\begin{array}{l}\text { Food category cost, } \\
\text { range }(\mathrm{NT} \$ / \mathrm{d}) \|\end{array}$} & & & \multicolumn{10}{|c|}{ Annual medical service utilisation $\dagger$} & \multicolumn{12}{|c|}{ Annual medical service costs $(1000 \mathrm{NT} \$) \ddagger \S$} \\
\hline & \multirow{2}{*}{\multicolumn{2}{|c|}{ All sample }} & \multicolumn{8}{|c|}{ Ambulatory care (visits) } & & & \multicolumn{8}{|c|}{ Ambulatory care } & \multirow{2}{*}{\multicolumn{2}{|c|}{$\begin{array}{l}\text { Hospitalisation } \\
\text { (d) }\end{array}$}} & \multirow{2}{*}{\multicolumn{2}{|c|}{ Total costs }} \\
\hline & & & \multicolumn{2}{|c|}{$\begin{array}{l}\text { Outpatient } \\
\text { services }\end{array}$} & \multicolumn{2}{|c|}{ Preventive care } & \multicolumn{2}{|c|}{ Dental services } & \multicolumn{2}{|c|}{ Emergency } & \multicolumn{2}{|c|}{$\begin{array}{l}\text { Hospitalisation } \\
\text { (d) }\end{array}$} & \multicolumn{2}{|c|}{$\begin{array}{l}\text { Outpatient } \\
\text { services }\end{array}$} & \multicolumn{2}{|c|}{$\underline{\text { Preventive care }}$} & \multicolumn{2}{|c|}{$\underline{\text { Dental services }}$} & \multicolumn{2}{|c|}{ Emergency } & & & & \\
\hline & Mean & Medians & Mean & Medians & Mean & Medians & Mean & Medians & Mean & Medians & Mean & Medians & Mean & Medians & Mean & Medians & Mean & Medians & Mean & Medians & Mean & Medians & Mean & Medians \\
\hline \&V (range) & & & $22 \cdot 7$ & $19 \cdot 4$ & 1.00 & 0.89 & 0.86 & 0.31 & 0.33 & 0.14 & 6.64 & $1 \cdot 21$ & $19 \cdot 3$ & 14.4 & 0.23 & 0.19 & 0.75 & 0.23 & 0.75 & 0.21 & 33.4 & 5.86 & $54 \cdot 3$ & 27.7 \\
\hline T1 $(<19.5)$ & $10 \cdot 7$ & $11 \cdot 0$ & $22 \cdot 2$ & $18 \cdot 6$ & 0.95 & 0.84 & 0.71 & 0.15 & 0.41 & 0.15 & 8.92 & 1.92 & $18 \cdot 1$ & $12 \cdot 7$ & 0.22 & 0.17 & 0.64 & 0.10 & 0.93 & 0.27 & $47 \cdot 7$ & $8 \cdot 13$ & 67.5 & $29 \cdot 7$ \\
\hline Т3 $(>38.6)$ & $61 \cdot 2$ & 54.7 & $24 \cdot 1$ & $21 \cdot 7^{\star \star \star}$ & 1.02 & 0.93 & 1.06 & $0.44^{\star \star \star}$ & 0.29 & $0.14^{\star \star \star}$ & 5.41 & $1.01^{\star \star \star}$ & $21 \cdot 2$ & $15 \cdot 9^{*}$ & 0.23 & 0.20 & 0.92 & $0.36^{\star}$ & 0.68 & $0.20^{*}$ & $26 \cdot 4$ & $5 \cdot 31^{\star \star \star}$ & 49.5 & $28 \cdot 7^{\star \star *}$ \\
\hline$P_{\text {trend }}$ & & & \multicolumn{2}{|c|}{$\star \star * \star$} & \multicolumn{2}{|c|}{ NS } & \multicolumn{2}{|c|}{ 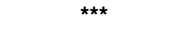 } & \multicolumn{2}{|r|}{ 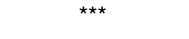 } & \multicolumn{2}{|r|}{ 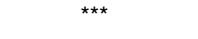 } & & * & & NS & & * & & * & & NS & & *** \\
\hline nimal derived (range) & & & & & & & & & & & & & & & & & & & & & & & & \\
\hline T1 $(<22.9)$ & $42 \cdot 3$ & $32 \cdot 1$ & 21.5 & $17 \cdot 1$ & 0.99 & 0.85 & 0.83 & 0.29 & 0.32 & 0.15 & 5.68 & 1.04 & $17 \cdot 7$ & $12 \cdot 8$ & 0.22 & 0.18 & 0.71 & 0.18 & 0.72 & 0.23 & 30.6 & $5 \cdot 17$ & $50 \cdot 0$ & 23.5 \\
\hline T2 (22.9-47.4) & $44 \cdot 1$ & 37.8 & $23 \cdot 1$ & $20 \cdot 1^{\star * \star}$ & 1.04 & 0.93 & 0.91 & 0.40 & 0.33 & 0.14 & 7.67 & $1 \cdot 19^{\star \star \star}$ & $18 \cdot 7$ & $15 \cdot 2$ & 0.24 & 0.20 & 0.77 & 0.26 & 0.71 & 0.18 & $35 \cdot 2$ & 5.91 & 55.5 & $28 \cdot 1$ \\
\hline T3 $(>47.4)$ & 38.7 & 32.9 & 23.5 & $20 \cdot 9^{\star \star \star}$ & 0.98 & 0.89 & 0.85 & 0.38 & 0.35 & 0.15 & 6.56 & $1.35^{\star \star \star}$ & $21 \cdot 4$ & $15 \cdot 7^{\star \star}$ & 0.23 & 0.19 & 0.75 & 0.23 & 0.81 & 0.23 & 34.3 & 6.73 & 57.5 & $30 \cdot 2^{*}$ \\
\hline$P_{\text {trend }}$ & & & & *** & & NS & & NS & & NS & & *** & & ** & & NS & & NS & & NS & & NS & & * \\
\hline train (range) & & & & & & & & & & & & & & & & & & & & & & & & \\
\hline $\mathrm{T} 1(<6.71)$ & 11.7 & 8.99 & 22.5 & 19.8 & 1.05 & 0.92 & 0.76 & 0.27 & 0.36 & 0.15 & 5.94 & 1.28 & 18.6 & 14.4 & 0.24 & 0.19 & 0.66 & 0.17 & 0.79 & 0.27 & 28.8 & 6.73 & 49.1 & $28 \cdot 0$ \\
\hline T3 $(>10.9)$ & $10 \cdot 8$ & $8 \cdot 15$ & $22 \cdot 9$ & 19.6 & 0.91 & $0.83^{*}$ & 0.91 & $0.41^{*}$ & 0.32 & 0.14 & $7 \cdot 11$ & $1 \cdot 23^{\star \star \star}$ & $20 \cdot 1$ & $15 \cdot 0$ & 0.20 & 0.17 & 0.78 & 0.26 & 0.68 & 0.20 & $35 \cdot 4$ & 5.45 & $57 \cdot 2$ & $28 \cdot 1^{*}$ \\
\hline$P_{\text {trend }}$ & & & & NS & & * & & * & & NS & & $\star \star \star \star ~$ & & NS & & NS & & NS & & NS & & NS & & * \\
\hline hiscellaneous (range) & & & & & & & & & & & & & & & & & & & & & & & & \\
\hline $\mathrm{T} 1(<0.48)$ & $6 \cdot 12$ & 0.80 & 23.0 & $18 \cdot 8$ & 0.97 & 0.85 & 0.84 & 0.29 & 0.37 & 0.15 & 6.85 & 1.52 & 18.4 & 13.9 & 0.22 & 0.18 & 0.75 & 0.19 & 0.81 & 0.25 & 33.3 & 7.77 & 53.5 & $29 \cdot 2$ \\
\hline T2 $(0.48-2.74)$ & $4 \cdot 34$ & 1.04 & $24 \cdot 0$ & $21 \cdot 5^{\star \star \star}$ & 1.03 & 0.95 & 0.86 & 0.39 & 0.31 & 0.14 & 6.99 & 1.07 & $20 \cdot 7$ & $15 \cdot 1$ & 0.23 & 0.20 & 0.75 & 0.24 & 0.69 & 0.19 & 35.7 & $5 \cdot 37$ & $58 \cdot 1$ & 26.8 \\
\hline T3 (>2.74) & $5 \cdot 13$ & 1.05 & $21 \cdot 2$ & $18 \cdot 1^{\star \star \star}$ & 1.00 & 0.88 & 0.88 & 0.32 & 0.32 & 0.15 & 6.07 & $1.07^{\star \star \star}$ & $18 \cdot 6$ & 14.4 & 0.23 & 0.19 & 0.74 & 0.26 & 0.74 & 0.22 & $31 \cdot 1$ & $5 \cdot 26$ & 51.4 & $26 \cdot 8$ \\
\hline$P_{\text {trend }}$ & & & & *** & & NS & & NS & & NS & & *** & & NS & & NS & & NS & & NS & & NS & & NS \\
\hline Ill food (range) & & & & & & & & & & & & & & & & & & & & & & & & \\
\hline T1 $(<69.7)$ & 70.1 & 63.8 & $21 \cdot 6$ & $17 \cdot 2$ & 0.98 & 0.85 & 0.75 & 0.27 & 0.34 & 0.14 & 7.05 & 1.23 & $17 \cdot 1$ & $12 \cdot 8$ & 0.22 & 0.17 & 0.64 & 0.15 & 0.74 & 0.21 & 36.6 & 5.75 & $55 \cdot 3$ & $23 \cdot 6$ \\
\hline T2 (69.7 - 102) & 88.5 & $81 \cdot 2$ & $22 \cdot 8$ & $19 \cdot 7^{\star \star *}$ & 1.02 & 0.91 & 0.81 & 0.29 & 0.35 & 0.14 & 7.40 & 1.27 & 18.5 & 14.5 & 0.23 & 0.20 & 0.72 & 0.21 & 0.82 & 0.21 & $36 \cdot 7$ & 6.76 & 57.0 & 28.9 \\
\hline T3 $(>102)$ & 116 & 110 & 23.7 & $21 \cdot 7^{\star \star \star}$ & 1.01 & 0.92 & 1.04 & $0.46^{\star \star \star}$ & 0.31 & 0.14 & 5.46 & $1 \cdot 15^{\star \star \star}$ & $22 \cdot 2$ & $16 \cdot 8^{\star \star \star}$ & 0.23 & 0.19 & 0.88 & 0.38 & 0.68 & 0.22 & $26 \cdot 7$ & $5 \cdot 01$ & $50 \cdot 7$ & $29 \cdot 3$ \\
\hline$P_{\text {trend }}$ & & & & 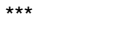 & & NS & & *** & & NS & & *** & & $\star \star \star \star ~$ & & NS & & * & & NS & & NS & & NS \\
\hline
\end{tabular}

$F \& V$, fruits and vegetables.

$P<0.05,{ }^{* *} P<0.01,{ }^{\star * \star} P<0.001$

† Outcome was assessed by GLM with Poisson distribution, T1 as a reference group.

₹ NTD, with an exchange rate about NTD 31 to USD 1 in 2015 .

$\S$ Outcome was assessed by GLM with $y$ distribution, $\mathrm{T} 1$ as a reference group.

$\|$ Food group costs were calculated as energy-adjusted food cost by residual method. 
Table 2. Participant characteristics by tertile (T) of daily fruits and vegetables (F\&V) costs (NT\$/d) ( $n$ 1445) (Numbers and percentages; mean values with their standard deviations)

\begin{tabular}{|c|c|c|c|c|c|c|c|c|}
\hline & \multirow[b]{3}{*}{$\%$ of sample } & \multicolumn{6}{|c|}{ Tertile of F\&V costs $(\mathrm{NT} \$ / \mathrm{d})^{*}$} & \multirow[b]{3}{*}{$P+$} \\
\hline & & \multicolumn{2}{|c|}{$\mathrm{T} 1$} & \multicolumn{2}{|c|}{$\mathrm{T} 2$} & \multicolumn{2}{|c|}{ T3 } & \\
\hline & & $n$ & $\%$ & $n$ & $\%$ & $n$ & $\%$ & \\
\hline$n, \%$ & 1445 & 482 & 33.4 & 482 & $33 \cdot 4$ & 481 & $33 \cdot 3$ & \\
\hline Sex (men \%) & 49.5 & $31 \cdot 3$ & & $33 \cdot 7$ & & $35 \cdot 0$ & & 0.224 \\
\hline Age (years, \%) & & & & & & & & \\
\hline $65-69$ & $44 \cdot 2$ & $31 \cdot 0$ & & $34 \cdot 3$ & & $34 \cdot 6$ & & $0 \cdot 132$ \\
\hline $70-74$ & $36 \cdot 4$ & 32.9 & & $34 \cdot 6$ & & 32.5 & & \\
\hline $75-79$ & $19 \cdot 5$ & 39.5 & & $28 \cdot 8$ & & $31 \cdot 7$ & & \\
\hline \multicolumn{9}{|l|}{ Ethnicity (\%) } \\
\hline Fukienese & $62 \cdot 7$ & $33 \cdot 3$ & & $36 \cdot 2$ & & $30 \cdot 5$ & & $<0.001$ \\
\hline Hakka & $10 \cdot 7$ & $26 \cdot 0$ & & $33 \cdot \overline{8}$ & & $40 \cdot 3$ & & \\
\hline Mainlander & $17 \cdot 3$ & $24 \cdot 1$ & & $28 \cdot 1$ & & $47 \cdot 8$ & & \\
\hline Indigenous & 9.42 & $58 \cdot 8$ & & $23 \cdot 5$ & & $17 \cdot 7$ & & \\
\hline Lived alone (\%) & $12 \cdot 8$ & $35 \cdot 5$ & & $28 \cdot 4$ & & $36 \cdot 2$ & & 0.399 \\
\hline \multicolumn{9}{|l|}{ Personal education (\%) } \\
\hline Illiterate & $32 \cdot 6$ & $37 \cdot 6$ & & $36 \cdot 7$ & & $25 \cdot 7$ & & $<0.001$ \\
\hline Primary and below & $46 \cdot 2$ & $34 \cdot 8$ & & $33 \cdot 0$ & & $32 \cdot 2$ & & \\
\hline Secondary & 14.7 & $26 \cdot 9$ & & $28 \cdot 3$ & & $44 \cdot 8$ & & \\
\hline Secondary and above & $6 \cdot 44$ & $17 \cdot 2$ & & $31 \cdot 2$ & & $51 \cdot 6$ & & \\
\hline \multicolumn{9}{|l|}{ Self-rated financial status (\%) } \\
\hline Enough & 14.9 & $20 \cdot 5$ & & $38 \cdot 1$ & & $41 \cdot 4$ & & $<0.001$ \\
\hline Just enough & $55 \cdot 2$ & $32 \cdot 0$ & & $33 \cdot 1$ & & 34.9 & & \\
\hline Some difficulty & $25 \cdot 2$ & $40 \cdot 7$ & & $32 \cdot 5$ & & $26 \cdot 8$ & & \\
\hline Very difficult & $4 \cdot 76$ & $52 \cdot 2$ & & $25 \cdot 4$ & & $22 \cdot 4$ & & \\
\hline \multicolumn{9}{|l|}{ Household income (NT\$/month, \%) } \\
\hline$<15000$ & $35 \cdot 9$ & 39.9 & & $35 \cdot 0$ & & $25 \cdot 2$ & & 0.310 \\
\hline $15000-29999$ & $19 \cdot 0$ & $35 \cdot 8$ & & $35 \cdot 1$ & & $29 \cdot 1$ & & \\
\hline $30000-49999$ & $21 \cdot 0$ & 33.5 & & $32 \cdot 3$ & & $34 \cdot 1$ & & \\
\hline$\geq 50000$ & $24 \cdot 1$ & $31 \cdot 8$ & & 33.9 & & 34.4 & & \\
\hline \multicolumn{9}{|l|}{ Perceived health status (\%) } \\
\hline Good & $40 \cdot 5$ & $26 \cdot 6$ & & $35 \cdot 4$ & & $38 \cdot 0$ & & $<0.001$ \\
\hline Fair & $46 \cdot 2$ & $36 \cdot 3$ & & $33 \cdot 7$ & & $30 \cdot 0$ & & \\
\hline Poor & $13 \cdot 3$ & $42 \cdot 1$ & & $25 \cdot 8$ & & $32 \cdot 1$ & & \\
\hline Charlson co-morbidity index & & & & & & & & $0.664 \ddagger$ \\
\hline Mean & 3.44 & 3.34 & & 3.45 & & 3.53 & & \\
\hline \multirow{2}{*}{\multicolumn{9}{|c|}{ Activities of daily life }} \\
\hline & & & & & & & & \\
\hline$\geq 1$ Difficulties & 0.83 & 0.63 & & 0.00 & & 0.21 & & 0.005 \\
\hline Physical activity (MET/d) & & & & & & & & $<0.001 \ddagger$ \\
\hline Mean & $2 \cdot 14$ & 1.62 & & $2 \cdot 33$ & & 2.47 & & \\
\hline SD & 3.47 & 3.38 & & 3.56 & & 3.43 & & \\
\hline$<1.5$ & 59.5 & 38.2 & & 31.5 & & $30 \cdot 3$ & & $<0.001$ \\
\hline $1 \cdot 5-3$ & $11 \cdot 0$ & 31.7 & & $37 \cdot 3$ & & $31 \cdot 0$ & & \\
\hline$>3$ & 29.5 & $24 \cdot 2$ & & 35.5 & & $40 \cdot 2$ & & \\
\hline \multicolumn{8}{|l|}{ Sleeping quality (\%) } & $0 \cdot 156$ \\
\hline Good & 31.4 & $29 \cdot 7$ & & $34 \cdot 2$ & & $36 \cdot 2$ & & \\
\hline Fair & $51 \cdot 5$ & $35 \cdot 7$ & & $31 \cdot 6$ & & $32 \cdot 8$ & & \\
\hline Poor & $17 \cdot 1$ & $32 \cdot 0$ & & $37 \cdot 3$ & & $30 \cdot 7$ & & \\
\hline Smoker (\%) & $21 \cdot 7$ & $23 \cdot 0$ & & 24.5 & & $17 \cdot 7$ & & 0.026 \\
\hline Alcohol drinker (\%) & $20 \cdot 8$ & $21 \cdot 2$ & & 21.9 & & $19 \cdot 3$ & & 0.603 \\
\hline Betel nut chewer & $7 \cdot 70$ & $11 \cdot 2$ & & $6 \cdot 46$ & & $5 \cdot 41$ & & 0.002 \\
\hline Unsatisfactory chewing ability (\%) & $34 \cdot 1$ & 35.5 & & $36 \cdot 3$ & & $28 \cdot 3$ & & 0.010 \\
\hline \multicolumn{9}{|l|}{ Dietary diversity scores (\%) } \\
\hline Mean & 4.46 & 3.84 & & $4 \cdot 63$ & & 4.92 & & \\
\hline SD & 1.06 & 1.07 & & 0.92 & & 0.88 & & \\
\hline$\leq 3$ & $16 \cdot 5$ & $65 \cdot 3$ & & $23 \cdot 0$ & & $11 \cdot 7$ & & $<0.001$ \\
\hline 4 & $32 \cdot 7$ & $43 \cdot 3$ & & 33.2 & & 23.5 & & \\
\hline 5 & $33 \cdot 8$ & $20 \cdot 3$ & & 37.0 & & $42 \cdot 7$ & & \\
\hline 6 & $16 \cdot 9$ & 9.02 & & 36.5 & & 54.5 & & \\
\hline
\end{tabular}

MET, metabolic equivalents.

${ }^{*}$ Food group costs were calculated as energy-adjusted food cost by residual method.

$\dagger$ Differences across T were tested using the $\chi^{2}$ test.

‡ Differences across T were tested using ANOVA. 
Table 3. Generalised linear model (GLM) results for annual medical service utilisation and costs by tertile (T) of fruits and vegetables (F\&V) and animalderived food costs (NT\$/d) ( $n$ 1445)

(exp ( $\beta$ Coefficients) and $95 \%$ confidence intervals)

\begin{tabular}{|c|c|c|c|c|c|c|c|c|}
\hline & \multicolumn{8}{|c|}{ Food costs (range NT\$/d) $\ddagger$} \\
\hline & \multicolumn{4}{|c|}{$\mathrm{F} \& \mathrm{~V}$} & \multicolumn{4}{|c|}{ Animal-derived foods } \\
\hline & \multicolumn{2}{|c|}{ T2 (19.6-38.7) } & \multicolumn{2}{|c|}{ T3 $(>38 \cdot 7)$} & \multicolumn{2}{|c|}{ T2 (23.0-47.4) } & \multicolumn{2}{|c|}{ T3 $(>47 \cdot 4)$} \\
\hline & $\exp (\beta)$ & $95 \% \mathrm{Cl}$ & $\exp (\beta)$ & $95 \% \mathrm{Cl}$ & $\exp (\beta)$ & $95 \% \mathrm{Cl}$ & $\exp (\beta)$ & $95 \% \mathrm{Cl}$ \\
\hline \multirow{2}{*}{\multicolumn{9}{|c|}{$\begin{array}{l}\text { Annual medical service utilisation§ } \\
\text { Ambulatory care (visits) }\end{array}$}} \\
\hline & & & & & & & \multicolumn{2}{|c|}{ Outpatient services } \\
\hline Model $1 \|$ & $\begin{array}{l}0.98 \\
1.02\end{array}$ & $\begin{array}{l}0.95,1.01 \\
0.99,1.05\end{array}$ & $\begin{array}{l}1.09^{\star \star \star} \dagger \\
1.13^{\star \star \star} \dagger\end{array}$ & $\begin{array}{l}1.05,1.13 \\
1.09,1.18\end{array}$ & $\begin{array}{l}1.09^{\star \star \star} \\
1.03^{\star}\end{array}$ & $\begin{array}{l}1.06,1.12 \\
1.00,1.07\end{array}$ & $\begin{array}{l}1.13^{\star \star \star \star} \dagger \\
1.07^{\star \star \star} \dagger\end{array}$ & $\begin{array}{l}1.08,1.17 \\
1.03,1.12\end{array}$ \\
\hline \multicolumn{9}{|l|}{ Emergency } \\
\hline $\begin{array}{l}\text { Model 1 } \\
\text { Model 2ף }\end{array}$ & $\begin{array}{l}0.73^{\star \star} \\
0.79\end{array}$ & $\begin{array}{l}0.58,0.91 \\
0.62,1.01\end{array}$ & $\begin{array}{l}0.79 \\
0.80\end{array}$ & $\begin{array}{l}0.59,1.05 \\
0.58,1.09\end{array}$ & $\begin{array}{l}1.03 \\
0.97\end{array}$ & $\begin{array}{l}0.81,1.31 \\
0.76,1.24\end{array}$ & $\begin{array}{l}1.26 \\
1.27\end{array}$ & $\begin{array}{l}0.91,1.73 \\
0.92,1.76\end{array}$ \\
\hline \multicolumn{9}{|c|}{ Hospitalisation (d) } \\
\hline Model $1 \|$ & $0.58^{\star \star \star}$ & $0.55,0.61$ & $\begin{array}{l}0.56^{\star *} \dagger \\
0.70^{\star * \star}+\end{array}$ & $0.53,0.60$ & $1.27^{\star \star \star}$ & $\begin{array}{l}1.21,1.34 \\
1.28 \\
1.43\end{array}$ & $\begin{array}{l}1 \cdot 12^{\star \star \star} \dagger \\
1.28^{\star * \star}+\end{array}$ & $\begin{array}{l}1.04,1.20 \\
1.191 .38\end{array}$ \\
\hline \multicolumn{9}{|c|}{$\begin{array}{l}\text { Annual medical service costs } \\
\text { (NT\$1000)†† } \\
\text { Outpatient services }\end{array}$} \\
\hline $\begin{array}{l}\text { Model } 1 \| \\
\text { Model } 24\end{array}$ & $\begin{array}{l}1.00 \\
1.02\end{array}$ & $\begin{array}{l}0.87,1.14 \\
0.90,1.17\end{array}$ & $\begin{array}{l}1 \cdot 10 \\
1 \cdot 12\end{array}$ & $\begin{array}{l}0.93,1.30 \\
0.95,1.32\end{array}$ & $\begin{array}{l}1.06 \\
1.03\end{array}$ & $\begin{array}{l}0.93,1.21 \\
0.90,1.17\end{array}$ & $\begin{array}{l}1 \cdot 18 \\
1 \cdot 15\end{array}$ & $\begin{array}{l}0.99,1.41 \\
0.96,1.37\end{array}$ \\
\hline \multicolumn{9}{|l|}{ Emergency } \\
\hline $\begin{array}{l}\text { Model 1 } \\
\text { Model 2ף }\end{array}$ & $\begin{array}{l}0.66^{\star \star} \\
0.73\end{array}$ & $\begin{array}{l}0.48,0.91 \\
0.52,1.03\end{array}$ & $\begin{array}{l}0.80 \\
0.80\end{array}$ & $\begin{array}{l}0.53,1.23 \\
0.51,1.25\end{array}$ & $\begin{array}{l}1.01 \\
0.88\end{array}$ & $\begin{array}{l}0.71,1.37 \\
0.61,1.26\end{array}$ & $\begin{array}{l}1.28 \\
1.24\end{array}$ & $\begin{array}{l}0.83,1.96 \\
0.77,1.98\end{array}$ \\
\hline \multicolumn{9}{|c|}{ Hospitalisation } \\
\hline Model $1 \|$ & $0.48^{\star \star \star}$ & $0.34,0.68$ & $0.51^{\star \star *}$ & $0.32,0.79$ & 1.18 & $0.81,1 \cdot 71$ & 1.51 & $0.94,2.44$ \\
\hline $\begin{array}{l}\text { Model 2ள } \\
\text { (I)tal medica }\end{array}$ & $0.66^{*}$ & $0.45,0.96$ & 0.66 & $0.41,1.06$ & 1.49 & $0.98,2.26$ & $1.83^{*} \dagger$ & $1 \cdot 06,3 \cdot 15$ \\
\hline \multicolumn{9}{|c|}{ Total medical costs } \\
\hline Model 24 & $0.78^{\star \star \star}$ & $0.67,0.91$ & $0.81^{*} \dagger$ & $\begin{array}{l}0.51,0.04 \\
0.67,0.98\end{array}$ & $1 \cdot 19^{\star}$ & $\begin{array}{l}0.94,1.29 \\
1.02,1.39\end{array}$ & $1.38^{\star \star} \dagger$ & $1.12,1.70$ \\
\hline
\end{tabular}

exp, exponentiate; $A D L$, activities of daily living; $\mathrm{CCl}$, Charlson co-morbidity index; DDS, dietary diversity score.

${ }^{\star} P<0.05,{ }^{\star \star} P<0.01,{ }^{\star \star \star} P<0.001$

$\dagger P_{\text {trend }}$ was significant.

‡ Food group costs were calculated as energy-adjusted food cost by residual method.

§ Outcome was assessed by GLM with Poisson distribution, T1 as a reference group.

|| Model 1: adjusted simultaneously for total food, fruits and vegetables and animal-derived food costs, age (years) and sex.

II Model 2: further adjusted for regions, ethnicity, personal education, living arrangement, self-rated financial status, household income (NT\$/month), perceived health status, ADL,

chewing ability, smoking, alcohol drinking, betel nut chewing, $\mathrm{CCI}$, DDS and physical activity.

†† Outcome was assessed by GLM with $\gamma$ distribution, T1 as a reference group.

\section{Discussion}

We found that higher F\&V and lower animal-derived food costs were associated with lower annual emergency (visits and costs), inpatient (days and costs) and total medical costs among community-dwelling elderly people. If older adults were to spend more on F\&V but less on animal-derived food, medical costs might be reduced.

F\&V consumption during older adulthood is associated with a reduced likelihood of critical chronic diseases ${ }^{(2)}$. Most studies have reported that a higher $\mathrm{F} \& \mathrm{~V}$ intake is protective against $\mathrm{CVD}^{(39,40)}$ and cancer ${ }^{(39,41)}$. In particular, large prospective cohort studies have reported that high $\mathrm{F} \& \mathrm{~V}$ consumption is associated with a low risk of all-cause, cancer and CVD mortalities ${ }^{(6,42,43)}$. F\&V are rich in fibre, antioxidants (e.g. vitamins $\mathrm{C}$ and $\mathrm{E}, \beta$ carotene and other carotenoids, Se, Zn, flavonoids and polyphenols), minerals (e.g. $\mathrm{K}, \mathrm{Mg}$ and $\mathrm{Ca}$ ), folate and phytochemicals ${ }^{(44-46)}$, and these may be some of the factors in combination that reduce the risk factors. Previous studies have argued that oxidative stress, through the initiation and progression of diseases such as CVD, hyperlipidaemia, hypertension, diabetes, cataracts, cancer, neurodegenerative diseases and ageing ${ }^{(44,47,48)}$, may be the most relevant mechanism involved; however, the pathways are likely to be multiple and complex. Moreover, the putative health effects of F\&V consumption are highly evident in the overall dietary pattern and depend on the synergistic action and mutual interaction of pluripotential food components ${ }^{(36,47,49)}$.

Although the health benefits of F\&V consumption are well documented, the economic costs involved have not been explored in detail ${ }^{(16,28)}$. Food cost has the potential to understand dietary affordability, relevant to many vulnerable groups such as the elderly participants - for example, the dietary recommendations made by the World Health Organization $^{(10)}$ encourage a daily consumption of five servings or a minimum of $400 \mathrm{~g}$ (excluding potatoes and other starchy tubers) of $\mathrm{F} \& \mathrm{~V}$ to reduce the risk of non-communicable diseases. The implementation of this recommendation would be more realistic if its consequences for food cost in relation to medical costs were known.

Daviglus et al. ${ }^{(39)}$ examined whether F\&V intake among middle-aged men in the USA was related to Medicare expenses in their old age and revealed that F\&V intakes were inversely associated with CVD- and cancer-related expenses as well as total expenses. The present study revealed that older adults 
(a)

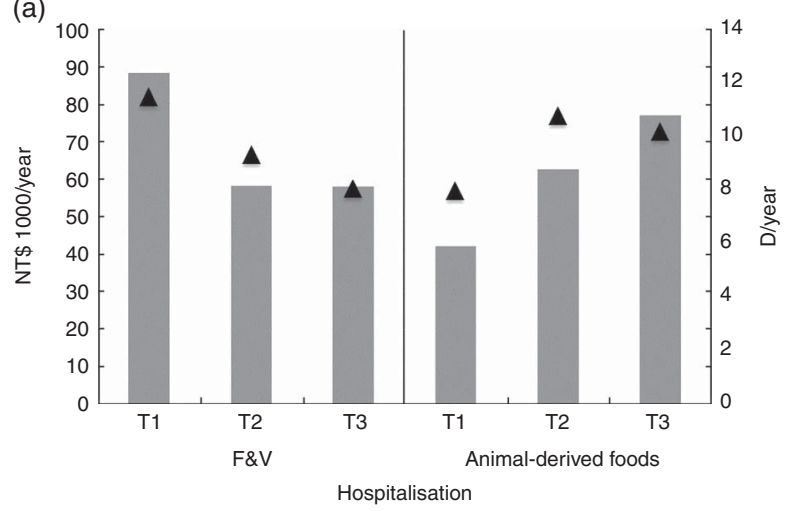

(b)

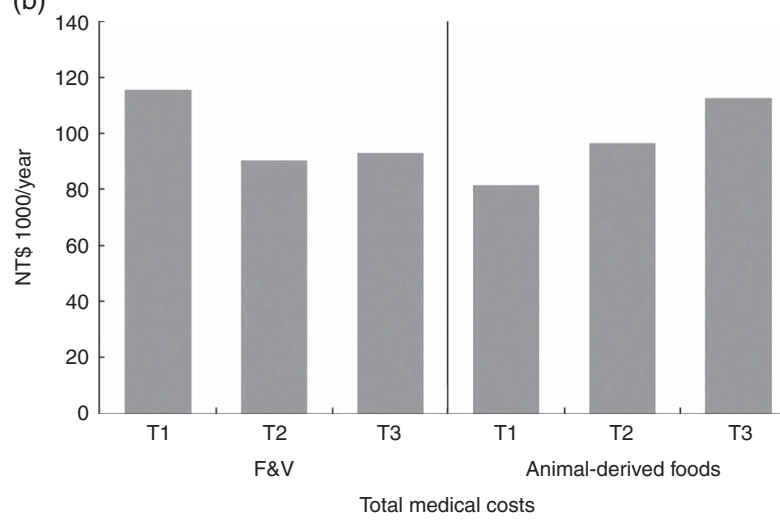

Fig. 2. Annual adjusted mean hospitalisation (days and costs) and total medical costs by tertiles $(T)$ of daily fruits and vegetable (F\&V) and animalderived food costs ( $n$ 1445). Models were adjusted simultaneously for total food, vegetable and fruit and animal-derived foods costs, age (years), sex, regions, ethnicity, personal education, living arrangement, self-rated financial status, household income (NT\$/month), perceived health status, activities of daily living, smoking status, alcohol drinking, betel nut chewing, chewing ability, Charlson co-morbidity index, dietary diversity score and physical activity. (a) $P_{\text {trends }}$ in F\&V ( $\mathbf{\Lambda}$, days: $P<0.001 ; \square$, costs: $P=0.062$ ); in animal-derived foods (days: $P<0.001$; costs: $P=0.032$ ). (b) $P_{\text {trends }}$ in F\&V (costs: $P=0.017$ ); in animal-derived foods (costs: $P=0.002$ )

who spent more on F\&V had fewer mean annual hospitalisation days, costs and total medical costs. For emergency visits and costs, the trends were similar, although not significant. The mean consumption of F\&V for T2 was $472 \mathrm{~g}$ and consistent with the WHO minimum recommendation. For both the USA (National Health and Nutrition Examination Survey; NHANES) ${ }^{(50)}$ and Taiwan (NAHSIT for 1993-1996, 1999-2000 and 2005-2008) data, there were only slight changes in F\&V intakes with adult age ${ }^{(51)}$. There may be scope to improve F\&V intakes in future by combining nutrition education with food price policies, which might contain support for F\&V systems ${ }^{(52)}$ and their accessibility.

In addition, the present study demonstrated that higher animal-derived food cost was associated with increased total medical costs. Animal-derived foods such as meat (red meat or processed meats) increase the risk of chronic diseases ${ }^{(53)}$, $\mathrm{CHD}^{(54)}$, type 2 diabetes $^{(55,56)}$, colorectal cancer ${ }^{(57)}$ and allcause mortality ${ }^{(58)}$. Nevertheless, small amounts of meat have the potential to improve protein and micronutrient intakes, (a)

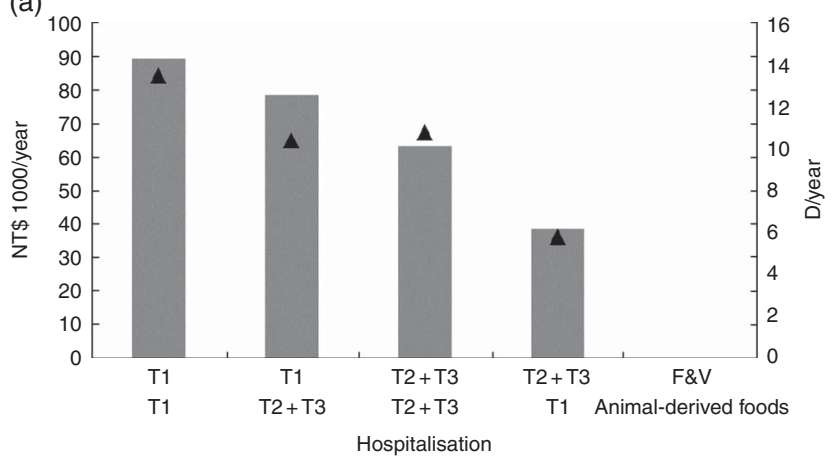

(b)

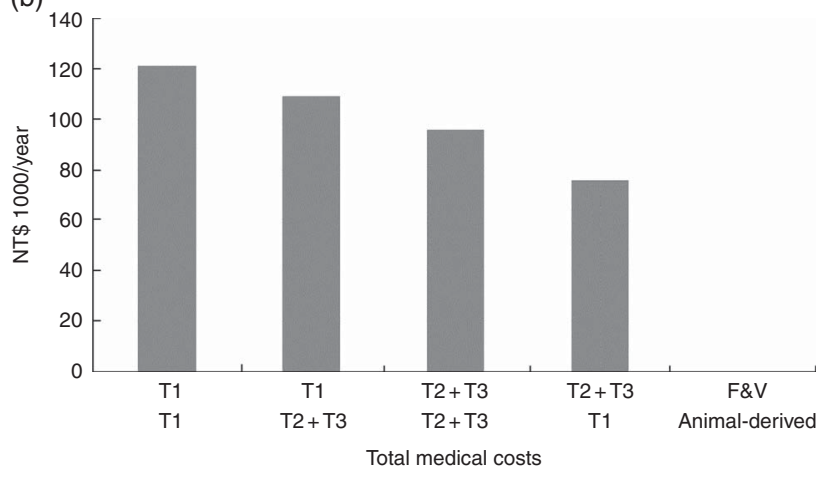

Fig. 3. Annual adjusted mean hospitalisation (days and costs) and total medical costs by combining daily fruits and vegetable (F\&V) and animalderived food costs (NT\$/d) ( $n$ 1445). Models were adjusted for total food costs, age (years), sex, regions, ethnicity, personal education, living arrangement, self-rated financial status, household income (NT\$/month), perceived health status, activities of daily living, smoking status, alcohol drinking, betel nut chewing, chewing ability, Charlson co-morbidity index, dietary diversity score and physical activity. $P_{\text {trends }}$ are all significant (a) $\mathbf{\Delta}$, Days: $P<0.001 ; \square$, costs: $P<0.001$; (b) costs: $P<0.001$ ).

through interaction with plant nutrients, and with the bioavailability of $\mathrm{Fe}^{(9)}$. Notably, poultry consumption has not been associated with all-cause mortality ${ }^{(9)}$. Odegaard et al. ${ }^{(59)}$ reported that dietary patterns rich in pork-based dim sum and meat were not associated with cancer or all-cause mortality in Chinese Singaporean non-smokers. Moreover, several vegetarian diet-based studies have not reported an increased all-cause mortality among meat eaters or non-vegetarians compared with vegetarians ${ }^{(60,61)}$; however, a rather modest benefit associated with CVD outcomes was observed ${ }^{(62)}$. In our study, the highest F\&V and the lowest animal-derived food cost group had the least hospitalisation days and lowest costs as well as total medical costs. This suggests that a food consumption pattern high in F\&V but low in animal-derived food could benefit health and reduce medical costs. As expected (see Table 4), older people with higher F\&V costs but low animal-derived food costs (F\&V, T2 + T3 and $\mathrm{AD}, \mathrm{T} 1)$ had lower intakes of fat, cholesterol and vitamin $\mathrm{D}$, but higher intakes of dietary fibre and vitamins $\mathrm{A}$ and $\mathrm{C}$, compared with those with other amounts of the combination of animal-derived and F\&V food costs. The dietary spending combination of this group had one of the lowest total food costs; yet, its quality was not unfavourable, except for vitamin $\mathrm{D}$ (and probably vitamin $\mathrm{B}_{12}$, although not 
Table 4. Daily energy and protein intakes and nutrient densities by tertile (T) of daily fruits and vegetables (F\&V) and animal-derived (AD) foods costs (NT\$/d) ( $n$ 1445) (Numbers and percentages; mean values)

\begin{tabular}{|c|c|c|c|c|c|c|c|c|c|c|c|}
\hline & & & \multicolumn{8}{|c|}{ Daily foods costs $(\mathrm{NT} \$ / \mathrm{d})^{*}$} & \multirow[b]{4}{*}{$P \dagger$} \\
\hline & & & \multirow{2}{*}{\multicolumn{2}{|c|}{$\begin{array}{l}F \& V(T 1) \\
A D(T 1)\end{array}$}} & \multirow{2}{*}{\multicolumn{2}{|c|}{$\begin{array}{c}F \& V(T 1) \\
A D(T 2+T 3)\end{array}$}} & \multirow{2}{*}{\multicolumn{2}{|c|}{$\frac{F \& V(T 2+T 3)}{A D(T 2+T 3)}$}} & \multirow{2}{*}{\multicolumn{2}{|c|}{$\frac{F \& V(T 2+T 3)}{A D(T 1)}$}} & \\
\hline & \multicolumn{2}{|c|}{ Total } & & & & & & & & & \\
\hline & $n$ & $\%$ & $n$ & $\%$ & $n$ & $\%$ & $n$ & $\%$ & $n$ & $\%$ & \\
\hline \multirow{2}{*}{\multicolumn{12}{|c|}{ Food costs $(\mathrm{NT} \$ / \mathrm{d})^{*}$}} \\
\hline & & & & & & & & & & & \\
\hline Total & 91.8 & & 43.4 & & $86 \cdot 7$ & & 113 & & $78 \cdot 7$ & & $<0.001$ \\
\hline $\mathrm{F} \& \mathrm{~V}$ costs $(\%$ of total) & 33.5 & $36 \cdot 4 \ddagger$ & $10 \cdot 4$ & 25.5 & $11 \cdot 0$ & $14 \cdot 0$ & $42 \cdot 9$ & $38 \cdot 1$ & 48.9 & $61 \cdot 2$ & $<0.001$ \\
\hline$A D$ costs $(\%$ of total) & 41.7 & $42.5^{\top}$ & $12 \cdot 0$ & 28.4 & 59.6 & $65 \cdot 8$ & 55.2 & 47.9 & 11.7 & $16 \cdot 0$ & $<0.001$ \\
\hline Energy intake $(\mathrm{kJ} / \mathrm{d})$ & 6807 & & 6468 & & 7033 & & 6937 & & 6502 & & \\
\hline Energy intake (kcal/d) & 1627 & & 1546 & & 1681 & & 1658 & & 1554 & & 0.029 \\
\hline \multicolumn{12}{|l|}{ Total protein intake } \\
\hline $\mathrm{g} / \mathrm{d}$ & $\begin{array}{l}68 \cdot 0 \\
16 \cdot 7\end{array}$ & & $\begin{array}{l}43 \cdot 1 \\
11 \cdot 2\end{array}$ & & $\begin{array}{l}75.5 \\
18.0\end{array}$ & & $\begin{array}{l}77 \cdot 2 \\
18 \cdot 6\end{array}$ & & $\begin{array}{l}55 \cdot 0 \\
14.2\end{array}$ & & $\begin{array}{l}<0.001 \\
<0.001\end{array}$ \\
\hline $\mathrm{g} / \mathrm{kg} \mathrm{BW/ \textrm {d }}$ & $1 \cdot 19$ & & 0.77 & & 1.31 & & 1.31 & & 0.99 & & $<0.001$ \\
\hline \multicolumn{12}{|c|}{ Protein intake by food category $(\mathrm{g} / \mathrm{d})$} \\
\hline Grain & $15 \cdot 9$ & & $19 \cdot 0$ & & $15 \cdot 8$ & & 14.7 & & $16 \cdot 6$ & & $<0.001$ \\
\hline $\mathrm{F} \& \mathrm{~V}$ foods & 10.8 & & 5.44 & & 4.92 & & 11.6 & & $18 \cdot 1$ & & $<0.001$ \\
\hline Vegetables & 5.46 & & 2.73 & & 3.00 & & 6.46 & & $7 \cdot 34$ & & $<0.001$ \\
\hline Fruits & 1.08 & & $0 \cdot 17$ & & 0.22 & & 1.45 & & 1.70 & & $<0.001$ \\
\hline Soyabean and products & $4 \cdot 24$ & & 2.54 & & 1.69 & & 3.65 & & 9.05 & & $<0.001$ \\
\hline$A D$ foods & 35.7 & & $12 \cdot 6$ & & $49 \cdot 3$ & & $45 \cdot 9$ & & 13.7 & & $<0.001$ \\
\hline Meats & $15 \cdot 2$ & & 5.44 & & 21.5 & & $19 \cdot 0$ & & $6 \cdot 21$ & & $<0.001$ \\
\hline Poultry and products & 3.41 & & 1.01 & & 4.93 & & 4.40 & & 1.14 & & $<0.001$ \\
\hline Livestock and products & 11.8 & & 4.43 & & $16 \cdot 5$ & & $14 \cdot 6$ & & 5.07 & & $<0.001$ \\
\hline Fish and shellfish & $12 \cdot 3$ & & 3.39 & & $18 \cdot 3$ & & $16 \cdot 2$ & & 3.11 & & $<0.001$ \\
\hline Eggs and products & 1.73 & & 1.38 & & 1.54 & & 1.90 & & 1.76 & & 0.267 \\
\hline Dairy products & 6.50 & & 2.40 & & 7.97 & & $8 \cdot 73$ & & $2 \cdot 61$ & & $<0.001$ \\
\hline \multicolumn{12}{|c|}{ Nutrient densities $(/ 4184 \mathrm{~kJ} / \mathrm{d}(1000 \mathrm{kcal} / \mathrm{d}))$} \\
\hline Protein $(\mathrm{g})$ & $42 \cdot 3$ & & 28.5 & & 46.5 & & $46 \cdot 9$ & & $36 \cdot 1$ & & $<0.001$ \\
\hline Fat $(\mathrm{g})$ & $27 \cdot 9$ & & $26 \cdot 6$ & & 30.4 & & 28.4 & & 24.8 & & $<0.001$ \\
\hline Cholesterol (mg) & 127 & & $75 \cdot 2$ & & 159 & & 146 & & $82 \cdot 2$ & & $<0.001$ \\
\hline Carbohydrate (g) & 143 & & 154 & & 130 & & 138 & & 160 & & $<0.001$ \\
\hline Dietary fibre $(\mathrm{g})$ & $12 \cdot 3$ & & 8.59 & & 7.00 & & 13.5 & & $16 \cdot 9$ & & $<0.001$ \\
\hline $\mathrm{Fe}(\mathrm{mg})$ & 7.44 & & 4.76 & & 6.44 & & $8 \cdot 13$ & & 8.51 & & $<0.001$ \\
\hline $\mathrm{Ca}(\mathrm{mg})$ & 414 & & 237 & & 394 & & 483 & & 386 & & $<0.001$ \\
\hline $\mathrm{Mg}(\mathrm{mg})$ & 145 & & 100 & & 123 & & 158 & & 167 & & $<0.001$ \\
\hline $\mathrm{Na}(\mathrm{mg})$ & 3083 & & 2504 & & 2773 & & 3227 & & 3415 & & $<0.001$ \\
\hline $\mathrm{K}(\mathrm{mg})$ & 1470 & & 826 & & 1177 & & 1712 & & 1613 & & $<0.001$ \\
\hline Vitamin A ( $\mu \mathrm{g} R E)$ & 747 & & 518 & & 492 & & 833 & & 948 & & $<0.001$ \\
\hline Vitamin D $(\mu \mathrm{g})$ & 3.77 & & 1.99 & & 4.48 & & 4.59 & & 1.95 & & $<0.001$ \\
\hline Vitamin $B_{1}(\mathrm{mg})$ & 0.68 & & 0.44 & & 0.65 & & 0.73 & & 0.73 & & $<0.001$ \\
\hline Vitamin $B_{2}(\mathrm{mg})$ & 0.85 & & 0.51 & & 0.86 & & 1.01 & & 0.70 & & $<0.001$ \\
\hline Niacin (mg) & 8.68 & & $6 \cdot 69$ & & 8.66 & & 9.26 & & 8.58 & & $<0.001$ \\
\hline Vitamin $B_{6}(\mathrm{mg})$ & 0.68 & & 0.45 & & 0.64 & & 0.77 & & 0.68 & & $<0.001$ \\
\hline Vitamin C (mg) & 97.8 & & 44.3 & & $49 \cdot 1$ & & 119 & & 132 & & $<0.001$ \\
\hline DDS & 4.46 & & 3.48 & & 4.05 & & 4.93 & & 4.43 & & $<0.001$ \\
\hline
\end{tabular}

BW, body weight; DDS, dietary diversity score

* Food costs were calculated as energy-adjusted food cost by residual method.

† Differences across T were tested by ANOVA.

$\mp$ The percentage of total food costs. 
assessed). Moreover, the total protein energy percentage was $14.2 \%$, which is acceptable and equates to $1 \mathrm{~g} / \mathrm{kg}$ body weight per $\mathrm{d}$. There are problems with protein intakes $(0.77 \mathrm{~g} / \mathrm{kg}$ body weight per d) where both F\&V and animal-derived expenditures are the lowest. Thus, a favourable diet need not be costly in order to save healthcare resources. Likewise, the amounts of food required to achieve these relatively favourable food and medical costs were modest.

Another possible explanation for these findings might be that those who purchase and eat less meat are more health conscious and have more favourable health outcomes - for example, older adults who spent more on F\&V and less on animal-derived foods had lower rates of smoking, alcohol drinking and betel nut chewing, but higher physical activity and better sleeping quality. Despite these covariates, the findings are unchanged. It also has to be noted that, in our study, when both F\&V and animal-derived food intakes are low, the medical costs are high, consistent with our previous findings that show a benefit of dietary diversity for medical costs ${ }^{(28)}$. Our findings are not associated with food avoidance, but with moderation of animal-derived food intake.

Our findings are in agreement with the WHO recommendations for F\&V consumption and draw attention to operationalising the recommendations regarding food category affordability and medical costs. In other words, public health nutrition policies require a nutritional-economic dimension.

\section{Limitations}

First, although our study was prospective, food group expenditure assessment was performed only at commencement. Thus, we have no information about changes in the eating behaviour or long-term dietary habits of the participants. However, community-dwelling elderly people have relatively stable diets ${ }^{(37,62)}$, and the reproducibility (intra-class correlation coefficient) for various nutrients of the three repeated 24-h dietary recalls for a subgroup population of fifty averaged $0 \cdot 46^{(63)}$. Second, food prices were derived from the national average monthly prices for Taiwan, and regional price variations were not considered ${ }^{(64)}$. Third, food prices were based on raw materials and did not include the costs of preparing food or eating out. This could have resulted in overestimation or underestimation of the actual food costs. Nevertheless, several studies have successfully used a similar food-pricing $\operatorname{approach}^{(12,16,28)}$. Finally, although the NHI covers $>99 \%$ of the population of Taiwan, out-of-pocket (OOP) payments are required for some medical-care services, pharmaceuticals and devices not covered by the NHI programme. In Taiwan, household income is positively associated with the use of healthcare and OOP payments ${ }^{(30)}$. OOP healthcare expenditures increase per household at all income levels; however, they constitute a substantial economic burden for low-income households $^{(31)}$. Therefore, the medical costs of high-income households may have been underestimated. Moreover, social disparities remain prevalent in healthcare accessibility and affordability, causing the medical service use and costs of low-income households to be underestimated. To address some of these limitations, we adjusted for SES and region.

\section{Conclusions}

F\&V cost is inversely associated with hospitalisation (days and costs) and total medical costs. Animal-derived food cost is positively associated with hospitalisation and medical costs among Taiwanese free-living older adults. A high F\&V and low animal-derived food spending pattern could lower the economic burden of the medical-care system.

\section{Acknowledgements}

The data sets used were obtained from the Elderly Nutrition and Health Survey in Taiwan, 1999-2000, conducted by the Center for Survey Research, Academia Sinica, and directed by Wen-Harn Pan and Su-Hao Tu. This study was partly based on data from the National Health Insurance Research Database provided by the Bureau of National Insurance, Department of Health, and managed by the National Health Research Institutes. The interpretation and conclusions of this study do not represent those of the Bureau of National Health Insurance, Department of Health or National Health Research Institutes.

Financial support for this project and for the research fellowship of Y.-T. C. L. was provided by the Ministry of Science and Technology (MOST103-2320-B-016-015-MY2, MOST103-2811B-016-003, MOST104-2811-B-016-007).

All the authors were involved in the study design and collectively wrote the paper; Y.-T. C. L. analysed the data; Y.-C. H. contributed to the interpretation of the results; M. L. W. and M.-S. L. coordinated the research; and M.-S. L. had the primary responsibility for the final content.

None of the authors has any conflicts of interest to declare.

\section{References}

1. Wang L, Manson JE, Gaziano JM, et al. (2012) Fruit and vegetable intake and the risk of hypertension in middle-aged and older women. Am J Hypertens 25, 180-189.

2. Hung HC, Joshipura KJ, Jiang R, et al. (2004) Fruit and vegetable intake and risk of major chronic disease. J Natl Cancer Inst $\mathbf{9 6 1}$, $577-1584$.

3. Sharma S, Vik S \& Kolonel LN (2014) Fruit and vegetable consumption, ethnicity and risk of fatal ischemic heart disease. J Nutr Health Aging 18, 573-578.

4. Nicklett EJ, Semba RD, Xue QL, et al. (2012) Fruit and vegetable intake, physical activity, and mortality in older communitydwelling women. J Am Geriatr Soc 60, 862-868.

5. Genkinger JM, Platz EA, Hoffman SC, et al. (2004) Fruit, vegetable, and antioxidant intake and all-cause, cancer, and cardiovascular disease mortality in a community-dwelling population in Washington County, Maryland. Am J Epidemiol 160, 1223-1233.

6. Zhang X, Shu XO, Xiang YB, et al. (2011) Cruciferous vegetable consumption is associated with a reduced risk of total and cardiovascular disease mortality. Am J Clin Nutr $\mathbf{9 4}$, 240-246.

7. Sinha R, Cross AJ, Graubard BI, et al. (2009) Meat intake and mortality: a prospective study of over half a million people. Arch Intern Med 169, 562-571.

8. Pan A, Sun Q, Bernstein AM, et al. (2012) Red meat consumption and mortality: results from 2 prospective cohort studies. Arch Intern Med 172, 555-563. 
9. Rohrmann S, Overvad K, Bueno-de-Mesquita HB, et al. (2013) Meat consumption and mortality - results from the European Prospective Investigation into Cancer and Nutrition. BMC Med 11, 63-75.

10. World Health Organization (2013) Global action plan for the prevention and control of noncommunicable diseases 2013-2020. http://apps.who.int/iris/bitstream/10665/94384/1/9789241506236_ eng.pdf?ua=1 (accessed April 2015).

11. Darmon N \& Drewnowski A (2008) Does social class predict diet quality? Am J Clin Nutr 87, 107-1117.

12. Lo YT, Chang YH, Lee MS, et al. (2012) Dietary diversity and food expenditure as indicators of food security in older Taiwanese. Appetite 58, 180-187.

13. Ho Chan WS (2010) Taiwan's healthcare report 2010. EPMAJ 1, 563-585.

14. Ministry of Health and Welfare (2012) National Health Expenditure Statistics 2012. http://www.mohw.gov.tw/EN/Ministry/Statistic.aspx?f_list_no=474andfod_list_no=3506 (accessed April 2015).

15. Campbell AA, Thorne-Lyman A, Sun K, et al. (2008) Greater household expenditures on fruits and vegetables but not animal source foods are associated with decreased risk of under-five child mortality among families in rural Indonesia. J Nutr 138, 2244-2249.

16. Lo YT, Chang YH, Wahlqvist ML, et al. (2012) Spending on vegetable and fruit consumption could reduce all-cause mortality among older adults. Nutr J 11, 113-122.

17. Pan WH, Hung YT, Shaw NS, et al. (2005) Elderly Nutrition and Health Survey in Taiwan (1999-2000): research design, methodology and content. Asia Pac J Clin Nutr 14, 203-210.

18. Wu SJ, Chang YH, Wei IL, et al. (2005) Intake levels and major food sources of energy and nutrients in the Taiwanese elderly. Asia Pac J Clin Nutr 14, 211-220.

19. Willett W (2013) Issues in analysis and presentation of dietary data. In Nutritional Epidemiology, 3rd ed. pp. 305-332 [W Willett, editor]. New York: Oxford University Press.

20. Yang Z, Norton EC \& Stearns SC (2003) Longevity and health care expenditures: the real reasons older people spend more. I Gerontol B Psychol Sci Soc Sci 58, S2-S10.

21. Fisheries Agency (1999-2000). Fish price query: Fisheries Agency, Agriculture and Food Agency, Council of Agriculture. Executive Yuan, Taipei, Taiwan. http://efish.fa.gov.tw/efish/statistics/ reportmap.htm (accessed April 2015).

22. Council of Agriculture (1999-2000). Yearly report of Taiwan's agriculture: Agriculture and Food Agency, Council of Agriculture. Executive Yuan, Taipei, Taiwan. http://www.coa.gov. tw/viewphp?catid=207 (accessed April 2015).

23. Council of Agriculture (1999-2000). Poultry and livestock products current trade: Agriculture and Food Agency, Council of Agriculture, Taipei, Taiwan. http://amis.afa.gov.tw/ (accessed April 2015)

24. Willett W (2013) Implications of total energy intake for epidemiologic analyses. In Nutritional Epidemiology, 3rd ed. pp. 260-286 [W Willett, editor]. New York: Oxford University Press.

25. Cheng TM (2009) Lessons from Taiwan's universal national health insurance: a conversation with Taiwan's Health Minister Ching-Chuan Yeh. Health Aff 281, 1035-1044.

26. Cheng TM (2003) Taiwan's new National Health Insurance program: genesis and experience so far. Health Aff (Millwood) 22, 61-76.

27. National Health Research Institutes (2015) National Health Insurance Research Database. http://nhird.nhri.org.tw/en/ Background.html (accessed April 2015).

28. Lo YT, Wahlqvist ML, Chang YH, et al. (2013) Dietary diversity predicts type of medical expenditure in elders. Am J Manag Care 19, e415-e 423.
29. Campbell SE, Seymour DG \& Primrose WR (2004) A systematic literature review of factors affecting outcome in older medical patients admitted to hospital. Age Ageing 33, 110-115.

30. Chi C, Lee JL, Tsai SL, et al. (2008) Out-of-pocket payment for medical care under Taiwan's National Health Insurance system. Health Econ 17, 961-975.

31. Sanmartin C, Hennessy D, Lu Y, et al. (2014) Trends in outof-pocket health care expenditures in Canada, by household income, 1997 to 2009. Health Rep 25, 13-17.

32. Moriya S, Tei K, Harada E, et al. (2011) Self-assessed masticatory ability and hospitalisation costs among the elderly living independently. J Oral Rehabil 38, 321-327.

33. Wiener JM, Hanley RJ, Clark R, et al. (1990) Measuring the activities of daily living: comparisons across national surveys. J Gerontol 45, S229-S237.

34. Huang YC, Wahlqvist ML \& Lee MS (2014) Appetite predicts mortality in free-living older adults in association with dietary diversity. A NAHSIT cohort study. Appetite 83, 89-96.

35. Huang YC, Wahlqvist ML \& Lee MS (2013) Sleep quality in the survival of elderly Taiwanese: roles for dietary diversity and pyridoxine in men and women. J Am Coll Nutr 32, 417-427.

36. Charlson ME, Pompei P, Ales KL, et al. (1987) A new method of classifying prognostic comorbidity in longitudinal studies: development and validation. J Chronic Dis 40, 373-383.

37. Lee MS, Huang YC, Su HH, et al. (2011) A simple food quality index predicts mortality in elderly Taiwanese. J Nutr Health Aging 15, 815-821.

38. Manning WG, Basu A \& Mullahy J (2005) Generalized modeling approaches to risk adjustment of skewed outcomes data. J Health Econ 24, 465-488.

39. Daviglus ML, Liu K, Pirzada A, et al. (2005) Relationship of fruit and vegetable consumption in middle-aged men to medicare expenditures in older age: the Chicago Western Electric Study. J Am Diet Assoc 105, 1735-1744.

40. Ellingsen I, Hjerkinn EM, Seljeflot I, et al. (2008) Consumption of fruit and berries is inversely associated with carotid atherosclerosis in elderly men. BrJ Nutr 99, 674-681.

41. Bradbury KE, Appleby PN \& Key TJ (2014) Fruit, vegetable, and fiber intake in relation to cancer risk: findings from the European Prospective Investigation into Cancer and Nutrition (EPIC). Am J Clin Nutr 100, 394s-398s.

42. Bellavia A, Larsson SC, Bottai M, et al. (2013) Fruit and vegetable consumption and all-cause mortality: a dose-response analysis. Am J Clin Nutr 98, 454-459.

43. Oyebode O, Gordon-Dseagu V, Walker A, et al. (2014) Fruit and vegetable consumption and all-cause, cancer and CVD mortality: analysis of Health Survey for England data. J Epidemiol Community Health 68, 856-862.

44. Uttara B, Singh AV, Zamboni P, et al. (2009) Oxidative stress and neurodegenerative diseases: a review of upstream and downstream antioxidant therapeutic options. Curr Neuropharmacol 7, 65-74.

45. Liu RH (2013) Health-promoting components of fruits and vegetables in the diet. Adv Nutr 4, 384s-392s.

46. Lako J, Trenerry VC, Wahlqvist ML, et al. (2007) Phytochemical flavonols, carotenoids and the antioxidant properties of a wide selection of Fijian fruit, vegetables and other readily available foods. Food Chem 101, 1727-1741.

47. Harasym J \& Oledzki R (2014) Effect of fruit and vegetable antioxidants on total antioxidant capacity of blood plasma. Nutrition 30, 511-517.

48. Knight JA (2000) Review: free radicals, antioxidants, and the immune system. Ann Clin Lab Sci 30, 145-158.

49. Trichopoulou A, Kouris-Blazos A, Wahlqvist ML, et al. (1995) Diet and overall survival in elderly people. BMJ $\mathbf{3 1 1}$, $1457-1460$. 
50. Casagrande SS, Wang Y, Anderson C, et al. (2007) Have Americans increased their fruit and vegetable intake? The trends between 1988 and 2002. Am J Prev Med 32, 257-263.

51. Wu SJ, Pan WH, Yeh NH, et al. (2011) Trends in nutrient and dietary intake among adults and the elderly: from NAHSIT 1993-1996 to 2005-2008. Asia Pac J Clin Nutr 20, 251-265.

52. Darmon N, Lacroix A, Muller L, et al. (2014) Food price policies improve diet quality while increasing socioeconomic inequalities in nutrition. Int J Behav Nutr Phys Act 11, 66.

53. von Ruesten A, Feller S, Bergmann MM, et al. (2013) Diet and risk of chronic diseases: results from the first 8 years of follow-up in the EPIC-Potsdam study. Eur J Clin Nutr 67, 412-419.

54. Micha R, Wallace SK \& Mozaffarian D (2010) Red and processed meat consumption and risk of incident coronary heart disease, stroke, and diabetes mellitus: a systematic review and meta-analysis. Circulation 121, 2271-2283.

55. Pan A, Sun Q, Bernstein AM, et al. (2011) Red meat consumption and risk of type 2 diabetes: 3 cohorts of US adults and an updated meta-analysis. Am J Clin Nutr 94, 1088-1096.

56. Kim Y, Keogh J \& Clifton P (2015) A review of potential metabolic etiologies of the observed association between red meat consumption and development of type 2 diabetes mellitus. Metabolism 64, 768-779.
57. Norat T, Bingham S, Ferrari P, et al. (2005) Meat, fish, and colorectal cancer risk: the European Prospective Investigation into Cancer and Nutrition. J Natl Cancer Inst 97, 906-916.

58. Larsson SC \& Orsini N (2014) Red meat and processed meat consumption and all-cause mortality: a meta-analysis. $\mathrm{Am} \mathrm{J}$ Epidemiol 179, 282-289.

59. Odegaard AO, Koh WP, Yuan JM, et al. (2014) Dietary patterns and mortality in a Chinese population. Am J Clin Nutr 100, $877-883$.

60. Key TJ, Appleby PN, Spencer EA, et al. (2009) Mortality in British vegetarians: results from the European Prospective Investigation into Cancer and Nutrition (EPIC-Oxford). $A m \mathrm{~J}$ Clin Nutr 89, 1613S-1619S.

61. Kwok CS, Umar S, Myint PK, et al. (2014) Vegetarian diet, Seventh Day Adventists and risk of cardiovascular mortality: a systematic review and meta-analysis. Int J Cardiol 176, 680-686.

62. Toffanello ED, Inelmen EM, Minicuci N, et al. (2010) Ten-year trends in dietary intake, health status and mortality rates in free-living elderly people. J Nutr Health Aging 14, 259-264.

63. Huang YC, Wahlqvist ML, Kao MD, et al. (2015) Optimal dietary and plasma magnesium statuses depend on dietary quality for a reduction in the risk of all-cause mortality in older adults. Nutrients 7, 5664-5683.

64. Clauson A \& McCray D (1997) Spotlight: food price changes vary regionally. Food Review 20, 5-7. 